\title{
Modelado molecular de los complejos de aluminio(III) con el péptido $\beta$-amiloide implicado en la enfermedad de Alzheimer
}

\author{
Adolfo E. Ensuncho ${ }^{1^{*}}$, Juana R. Robles ${ }^{1}$ y Jennifer J. Lafont ${ }^{2}$ \\ (1) Facultad de Ciencias, Dpto. de Química, Universidad de Córdoba, Grupo de Química Computacional, Cra, 6 \#76-305, \\ Montería-Colombia. (correo-e: aensuncho@correo.unicordoba.edu.co, jrobles@correo.unicordoba.edu.co) \\ (2) Facultad de Ciencias, Dpto. de Química, Universidad de Córdoba, Grupo de Fisicoquímica orgánica y Biocombustibles, \\ Cra, 6 \#76-305, Montería-Colombia. (correo-e: jlafont@correo.unicordoba.edu.co)
}

Recibido Ene. 13, 2020; Aceptado Mar. 9, 2020; Versión final May. 18, 2020, Publicado Oct. 2020

\begin{abstract}
Resumen
Se estudiaron computacionalmente las propiedades geométricas, electrónicas y termodinámicas de un grupo de complejos formados por aluminio(III) con algunos aminoácidos (AA) presentes en la región de afinidad por metales del péptido $\beta$-amiloide ( $\beta$-A) (1-16), implicado en la enfermedad de Alzheimer. Para los cálculos computacionales de los complejos, se usó el funcional híbrido B3LYP en conjunto con la base $6-311+G(d, p)$. Las geometrías obtenidas correspondieron a complejos tetra-coordinados (CT) y penta-coordinados (CP). Se encontraron interacciones de aluminio(III) con los átomos de $\mathrm{O}$ y $\mathrm{N}$ de los ligandos fenolato (Ph-O), carboxilato $\left(\mathrm{COO}^{-}\right)$, carbonilo $(\mathrm{C}=\mathrm{O})$, amínicos $(\mathrm{RN})$ e imidazólicos $(\mathrm{ImN})$. De acuerdo con el análisis de orden natural de enlace, las interacciones con mayor fortaleza de enlace fueron las encontradas para los ligandos fenolato e imidazólico. En conclusión, el análisis termodinámico de las estructuras estudiadas mostró que los complejos penta-coordinados tienden a ser más estables que los tetra-coordinados.
\end{abstract}

Palabras clave: enfermedad de Alzheimer; péptido $\beta$-amiloide; aluminio(III); cálculos DFT; complejos tetra y penta-coordinados

\section{Molecular modeling of the aluminum(III) complexes with the $\beta$ - amyloid peptide involved in Alzheimer's disease}

\begin{abstract}
This research study examined computationally the geometric, electronic, and thermodynamic properties of a group of complexes formed by aluminum(III) and some amino acids present in the $\beta A$ peptide, which are involved in the development of Alzheimer's disease. Density Functional Theory (DFT) was used for modeling complexes at the level of theory B3LYP/6-31+G(d,p) resulting in five-coordinated $(C P)$ and four-coordinated (CT) compounds. There were aluminum(III) interactions with $\mathrm{O}^{-}$and $\mathrm{N}$ ligands: phenolate ( $\mathrm{Ph}^{-} \mathrm{O}^{-}$), carboxylate $\left(\mathrm{COO}^{-}\right)$, carbonyl $(\mathrm{C}=\mathrm{O})$, amino $(\mathrm{RN})$, and imidazole $(\mathrm{ImN})$. The interactions with greater strength were phenolate and imidazole. In conclusion, the thermodynamic analyses of the structures examined showed that the five-coordinated complexes tend to be more stable than the four-coordinated complexes.
\end{abstract}

Keywords: Alzheimer's disease; $\beta$-amyloid peptide; aluminum(III); DFT calculations; four- and five-coordinated complexes 


\section{INTRODUCCIÓN}

La enfermedad de Alzheimer es un tipo de demencia progresiva asociada a procesos neurodegenerativos que inducen disfunción cognitiva y trastornos conductuales en personas de edad avanzada (Nakano et al., 2015; Weintraub et al., 2012). Esta problemática de salud es de impacto mundial, ya que, en 2010 se estimó un número cercano a 36 millones de personas con demencia por Alzheimer en el mundo, y se proyecta que esta cantidad se duplicará para el año 2030 y superará el triple para el 2050 (Brookmeyer, et al., 2007).

La característica principal observada en el cerebro de los pacientes con esta patología son las placas seniles que están formadas por depósitos extracelulares del péptido $\beta$-amiloide y por ovillos neurofibrilares. En general, los péptidos $\beta$-amiloide constituyen una familia de moléculas que contienen secuencias peptídicas de 39 a 43 aminoácidos, donde la secuencia más toxica corresponde a la formada por 42 aminoácidos $\beta A(1-$ 42) (Provias y Jeynes, 2014). Si bien la agregación de los péptidos $\beta$-amiloides es un aspecto clave que desencadena los eventos degenerativos que causan el daño neuronal característico de la enfermedad de Alzheimer (CSerpell, 2000; Platts, 2020), la importancia y participación de iones metálicos en este proceso es cada vez más reconocida (Kepp, 2017). Así, por ejemplo, estudios recientes revelan que algunos metales como $\mathrm{Zn}^{2+}, \mathrm{Cu}^{2+}$, y $\mathrm{Fe}^{2+}$ promueven la agregación de los $\beta$-amiloides mediante procesos de óxidaciónreducción, (Bonda et al., 2011; Platts, 2020). Adicionalmente, el análisis post-mortem en cerebros de pacientes con Alzheimer, se han encontrado concentraciones apreciables de estos iones, lo que sugiere que los metales tienen un rol importante en el desarrollo de la enfermedad de Alzheimer (Bush, 2003; Mujika et al., 2017). En conexión con lo expuesto, se ha establecido que algunas interacciones moleculares metal- $\beta A(1-$ 42) son responsables de acelerar la agregación $\beta A$ (Drew y Barnham, 2011; Rauk, 2009; Tõugu, et al., 2011) y se ha demostrado que la región $\beta A(1-16)$, presenta gran afinidad hacia los metales (Strodel y CoskunerWeber, 2019), lo que ha despertado interés en la investigación de este mecanismo debido a que existe la posibilidad de utilizar dichos iones como posibles dianas terapéuticas, así como también, comprender los procesos de quelación de estos iones metálicos.

Las investigaciones en esta dirección, se han enfocado principalmente en los metales $\mathrm{Zn}$ (II), $\mathrm{Cu}$ (II) y Fe(II) (Mujika et al., 2017). No obstante, los estudios durante varias décadas han implicado al Al(III) en la aparición de la enfermedad de Alzheimer (Platts, 2020). Así por ejemplo, estudios anteriores realizados por Exley et al. (1993), mostraron que el $\mathrm{Al}(\mathrm{III})$ tiene la capacidad de unirse al péptido $\beta$-amiloide afectando su conformación y promoviendo la agregación. Por su parte, Mujika et al. (2011), reportó que la interacción de Al(III) con el péptido permite la formación de especies reactivas de oxígeno. Posteriores estudios mediante espectroscopia de fluorescencia con Al(III) y el péptido $\beta \mathrm{A}(1-42)$ mostraron el mismo comportamiento (Harrington, et al., 2011; Bolognin et al., 2011). También, Zhang et al. (2019), demostraron mediante técnicas de dicroísmo circular y espectroscopia de fuerza atómica que $\mathrm{Al}(\mathrm{III})$ induce la transformación del péptido $\beta A(1-40)$ desde su conformación inicial enrollada aleatoria a la configuración de $\beta$-lámina. Por tanto, la interacción de aluminio(III) con polipéptidos es un tema de elevada importancia, dado que es una característica central para comprender sus efectos nocivos en los sistemas biológicos. Ahora bien, aunque en la literatura científica se reportan estudios experimentales de la interacción de $\mathrm{Al}(\mathrm{III})$ con $\beta \mathrm{A}$, las investigaciones computacionales para estos sistemas moleculares son escasas (Strodel y Coskuner-Weber, 2019). Una serie de estudios basados en la teoría de los funcionales de la densidad (DFT, por sus siglas en inglés) examinaron la capacidad del aluminio(III) para unirse con algunas biomoléculas (Mujika et al., 2014), y también su actividad pro-oxidante (Mujika et al., 2011).

En un estudio reciente, Mujika et al. (2018), plantearon que las interacciones se establecen principalmente mediante la unión del aluminio a cadenas laterales de aminoácidos fosforiladas y no fosforiladas. Sin embargo, recientemente se ha propuesto un nuevo paradigma estructural, en el que el aluminio interactúa directamente con la columna vertebral de las proteínas, provocando cambios drásticos en su estructura secundaria y conduciendo finalmente a su desnaturalización. En dicho estudio, los resultados muestran la preferencia de aluminio(III) hacia las cadenas laterales de aminoácidos, en lugar del esqueleto peptídico (Mujika et al., 2018).

Otras investigaciones recientes basadas en la DFT, buscan caracterizar el entorno de coordinación de $\mathrm{Al}(\mathrm{III})$ con $\beta A$, ya que esto es una pieza clave para entender las interacciones que ocurren en detalle molecular del metal con el péptido (Thaís et al., 2013). En este sentido, la química computacional surge como una valiosa herramienta que complementa los resultados experimentales, $y$ al mismo tiempo provee de un conocimiento adicional sobre la unión entre el péptido $\beta A$ con el ion metálico $\mathrm{Al}(\mathrm{III})$. De acuerdo con lo anterior, es claro que resulta interesante estudiar el entorno de coordinación de aluminio(III) debido al papel clave que tiene en la agregación del péptido. En consecuencia, el presente estudio computacional tiene como objetivo determinar las interacciones de aluminio(III) con algunos aminoácidos de la región de afinidad por metales del péptido $\beta A(1-16)$, con el fin de obtener información valiosa que permita comprender las interacciones de este metal con biomoléculas que participan en la enfermedad de Alzheimer. 


\section{METODOLOGÍA}

Los cálculos de estructura electrónica se llevaron a cabo mediante el paquete computacional Gaussian03 (Frisch et al., 2003), usando la Teoría del Funcional de la Densidad (DFT, por sus siglas en inglés) (Kohn et al., 1996) y el método de Hartree-Fock (Slater, 1951). Inicialmente, se llevaron a cabo cálculos de optimización geométrica y frecuencias mediante los funcionales B3LYP y M06-2X en conexión con la base 6-311+G(d,p). Para determinar el entorno de coordinación del ion $\mathrm{Al}$ (III) se tuvo en cuenta la energía libre de hidratación, siguiendo el esquema reportado por Ali-Torres et al. (2015). La energía libre de hidratación se expresa mediante la ecuación (1),

$$
\Delta \mathrm{G}=\Delta \mathrm{G}_{\mathrm{C}}-\left(\Delta \mathrm{G}_{\mathrm{Al}(\mathrm{III})}+\mathrm{n} \Delta \mathrm{G}_{\mathrm{H}_{2} \mathrm{O}}\right)
$$

En donde $\Delta \mathrm{G}_{c}$, representa la energía libre molar del complejo $\mathrm{Al}(\mathrm{III})-\left(\mathrm{H}_{2} \mathrm{O}\right) \mathrm{n},(\mathrm{n}=1,2, \ldots, 6), \Delta \mathrm{G}_{\mathrm{Al}(\mathrm{III})}$ es la energía libre molar del catión $\mathrm{Al}(\mathrm{III})$ y finalmente $\mathrm{nG}_{\mathrm{H}_{2} \mathrm{O}}$ es la energía libre molar de las moléculas de agua presentes en el complejo. Subsecuentemente, se utilizaron los complejos más estables $(\Delta G<0)$ para determinar el número de ligandos que podrían unirse al ion $\mathrm{Al}(\mathrm{III})$. El aluminio(III), puede formar una gran variedad de especies hidratadas (Yang et al., 2009). Seguidamente, la construcción de los modelos moleculares Al(III)- $\beta A$ se realizó mediante la interfaz del programa informático Avogadro (Hanwell et al., 2012). Se escogieron los grupos - $\mathrm{R}$ de los aminoácidos (designados como AA); acido aspártico 1 (Asp1), alanina 2 (Ala2), ácido glutámico 3 (Glu3), histidina6 (His6), tirosina 10 (Tyr10), ácido glutámico 11 (Glu11), histidina 13 (His13), e histidina 14 (His14), como los sitios de coordinación de $\mathrm{Al}(\mathrm{III})$ y que se encuentran en la región de afinidad por metales del péptido $\beta A$ (Strodel y Coskuner-Weber, 2019). También, se tuvieron en cuenta ligandos pertenecientes a enlaces peptídicos, ya que ambos han sido reportados en estudios anteriores como posibles sitios de unión metal- $\beta \mathrm{A}$ (figura 1). Las estructuras de los modelos $\mathrm{Al}(\mathrm{III})$-AA construidos en el paso anterior, fueron inicialmente optimizadas con el modelo químico HF/6-31G. Posteriormente, los complejos que convergieron a mínimos de energía se optimizaron nuevamente con el funcional B3LYP y la base 6$311+G(d, p)$. Cabe mencionar que los complejos con frecuencias negativas fueron descartados en la realización de este estudio.

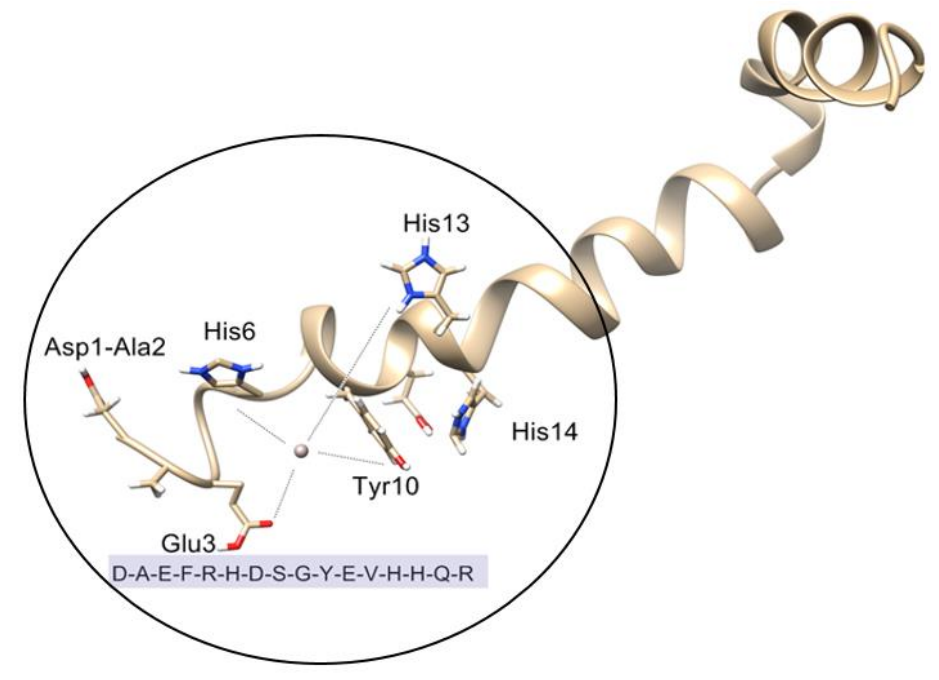

Fig.1. Región de afinidad por metales del péptido $\beta$-Aamiloide(1-16)

Una vez obtenidos los mínimos de energía para los complejos Al(III)-AA, se discutió su estabilidad termodinámica con base en el cambio de la energía libre de formación (ecuación 2).

$$
\Delta \mathrm{G}=\Delta \mathrm{G}_{\mathrm{Al}(\mathrm{III})-\mathrm{AA}}-\left(\Delta \Delta_{\mathrm{Al}(\mathrm{III})}+\Delta \mathrm{G}_{\mathrm{AA}}\right)
$$

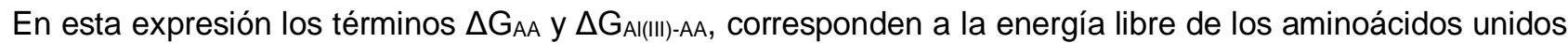
a $\mathrm{Al}(\mathrm{III})$ y la energía libre del complejo $\mathrm{Al}(\mathrm{III})-\mathrm{AA}$, respectivamente. Si el valor de $\Delta \mathrm{G}<0$, indica que la unión de dichos aminoácidos al ion $\mathrm{Al}(\mathrm{III})$ se ve favorecida termodinámicamente. También se tuvieron en cuenta las correcciones de energía del punto cero (Figueredo, Ensuncho, y López, 2014) y el error por superposición del conjunto base (BSSE, por sus siglas en inglés) usando el método counterpoise (Lopes et al., 2010). La fortaleza relativa de las interacciones AI(III)-AA se analizaron mediante el índice de enlace de Wiberg, el cual se calculó mediante el programa NBO (Mottishaw et al., 2015). Cabe resaltar que el análisis NBO es una herramienta eficiente en los cálculos de análisis de población, ya que es más robusto que el enfoque tradicional de Mulliken. Si bien el programa NBO es casi insensible al cambio del conjunto de funciones base 
a diferencia del análisis de población de Mulliken, el cual es altamente sensible a dichos cambios otra ventaja del análisis NBO es que proporciona una representación localizada de la densidad electrónica sobre una molécula, lo que la hace una aproximación más realista y ajustada a la intuición química.

\section{RESULTADOS Y DISCUSIÓN}

En la tabla 1, se muestran los valores de $\Delta \mathrm{G}$ de hidratación calculados para los complejos $\mathrm{Al}(\mathrm{III})-\left(\mathrm{H}_{2} \mathrm{O}\right)_{\mathrm{n}}$, (donde $n=1,2, \ldots, 6$ ). Como se observa en la tabla 1 , para ambos funcionales se mantiene la tendencia energética. En cuanto a los entornos de coordinación, los de mayor estabilidad corresponden a $n=4$ y $n=5$, ya que mostraron los valores más negativos de $\Delta \mathrm{G}$. La estabilidad del complejo penta-coordinado ya se había reportado en trabajos anteriores donde se mostró que este arreglo espacial de átomos interactuaba fuertemente con polipéptidos modelo (Son et al., 2014). Asimismo, Swaddle et al. (2005), mostraron experimentalmente que las especies penta-hidratadas de este ion pueden existir a nivel biológico en un rango de $\mathrm{pH}$ entre 4.3 a 7.0 aproximadamente. Adicionalmente, se observa que la hexa-coordinación del Al(III) no se ve favorecida termodinámicamente debido a que $\Delta G>0$. Las geometrías que exhiben estos complejos ( $n$ $=4,5,6$ ) se muestra en la figura 2 . El complejo Al-[H $\mathrm{H}_{2} \mathrm{O}_{4}$ presenta una geometría tetraédrica, el complejo Al$\left[\mathrm{H}_{2} \mathrm{O}_{5}\right.$ una geometría bipiramidal-trigonal y el complejo $\mathrm{Al}-\left[\mathrm{H}_{2} \mathrm{O}\right]_{6}$ una geometría octaédrica. La formación de las especies penta y hexacoordinada ya había sido reportado en la literatura científica (Yang et al., 2009).

Tabla 1: Energía libre de Gibbs de los complejos Al(III)-[ $\left[\mathrm{H}_{2} \mathrm{O}\right]_{n} \quad(n=1,2 \ldots ., 6)$ en $\mathrm{kcal} \mathrm{mol}^{-1}$

\begin{tabular}{|c|c|c|}
\hline Complejo & $\Delta G(\mathrm{~B} 3 \mathrm{LYP})$ & $\Delta G(\mathrm{M} 06-2 \mathrm{X})$ \\
\hline $\mathrm{Al}-\left[\mathrm{H}_{2} \mathrm{O}\right]_{5}$ & $-593,426$ & $-616,313$ \\
\hline $\mathrm{Al}-\left[\mathrm{H}_{2} \mathrm{O}\right]_{4}$ & $-549,439$ & $-564,085$ \\
\hline $\mathrm{Al}-\left[\mathrm{H}_{2} \mathrm{O}\right]_{3}$ & $-466,153$ & $-473,374$ \\
\hline $\mathrm{Al}-\left[\mathrm{H}_{2} \mathrm{O}\right]_{2}$ & $-352,730$ & $-355,110$ \\
\hline $\mathrm{Al}-\left[\mathrm{H}_{2} \mathrm{O}\right]$ & $-195,871$ & $-195,226$ \\
\hline $\mathrm{Al}-\left[\mathrm{H}_{2} \mathrm{O}\right]_{6}$ & 1892,386 & 1816,856 \\
\hline
\end{tabular}

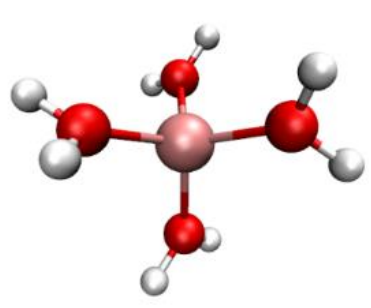

$\mathrm{Al}-\left[\mathrm{H}_{2} \mathrm{O}\right]_{4}$

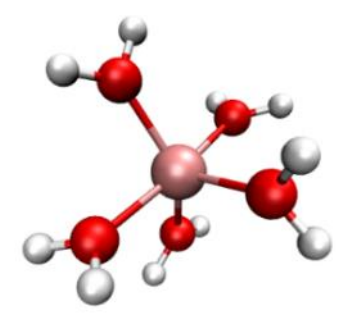

$\mathrm{Al}-\left[\mathrm{H}_{2} \mathrm{O}\right]_{5}$

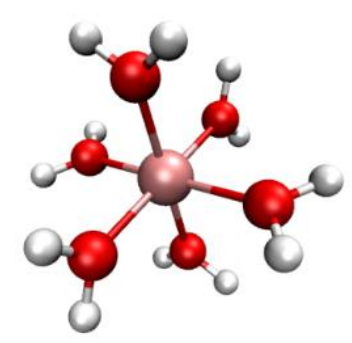

$\mathrm{Al}-\left[\mathrm{H}_{2} \mathrm{O}\right]_{6}$

Fig. 2. Geometrías optimizadas de los complejos Al-[H2O $]_{n}(n=4,5,6)$

Los entornos de coordinación de $\mathrm{Al}(\mathrm{III})$ favorecidos termodinámicamente corresponden a aquellos con cuatro (CT) y cinco ligandos (CP), respectivamente. Los motivos geométricos de los complejos se muestran en la figura 3. En la estructura de cada complejo se observa la presencia de interacciones O-Al(III), N-Al(III) y C$\mathrm{Al}(\mathrm{III})$, donde, los complejos tetra-coordinados (CT) poseen en su mayoría enlaces tipo O-Al(III). En nuestro caso se observó la preferencia por los grupos carboxilato y fenolato, respectivamente. En general, se encontró que la distancia O-Al(III) en los enlaces tipo Ph-O- $(1,70 \AA \hat{A}-1,75 \AA \AA)$ es menor en comparación con los tipos $\mathrm{C}=\mathrm{O}$ y $\operatorname{COO}^{-}(1,83 \AA \hat{A}-1,95 \AA \hat{)})$, sugiriendo que la interacción con el aminoácido Tyr10 es más fuerte con respecto a otros ligandos oxigenados, lo cual es consistente con trabajos computacionales previos (Mujika et al., 2014; Mujika et al., 2017). Mujika et al. (2018), ya había sugerido que las estructuras en las que el aluminio interactúa con cadenas laterales cargadas negativamente como el ácido aspártico son altamente favorecidas termodinámicamente. Por otra parte, las interacciones $\mathrm{N}$-Al(III) son más frecuentes en complejos pentacoordinados $(\mathrm{CP})$ debiéndose principalmente a ligandos imidazólicos $(\operatorname{ImN})$ con distancias de enlace que varían entre $1,88 \hat{\text { y }} 2,03 \hat{A}$ y ligandos amínicos (RN), con distancias de $2,01 \hat{\text { a }} 2,13 \hat{A}$, respectivamente. Las interacciones O-AI(III) y N-Al(III) incluyen la participación de los residuos His6, His13, His14, Tyr10 presentes 
en el péptido $\beta A$ y también los $N$ peptídicos de los residuos Asp1, Ala2, His13, indicando que estos sitios están involucrados en la agregación del péptido $\beta A$, lo cual está en buen acuerdo con lo reportado previamente por Mujika et al. (2014).

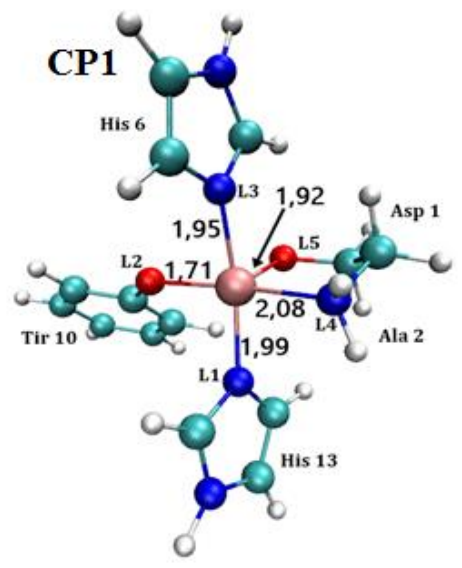

CT1

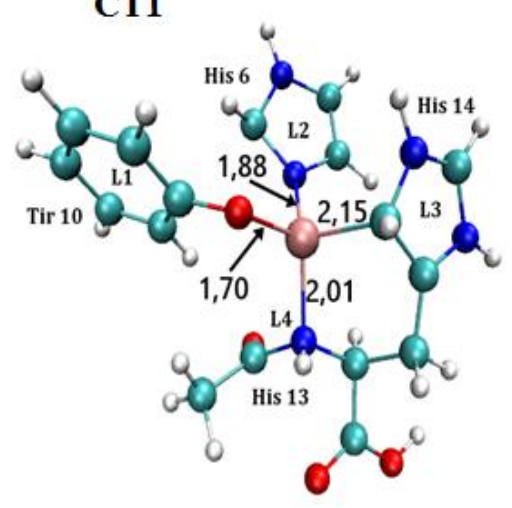

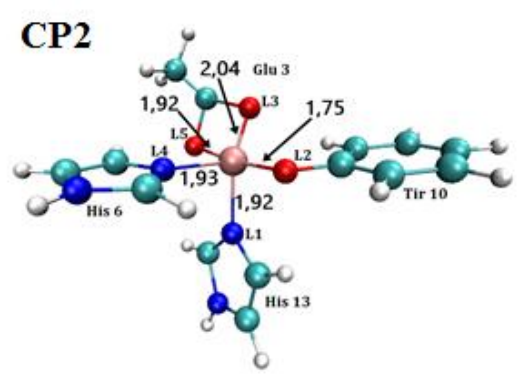

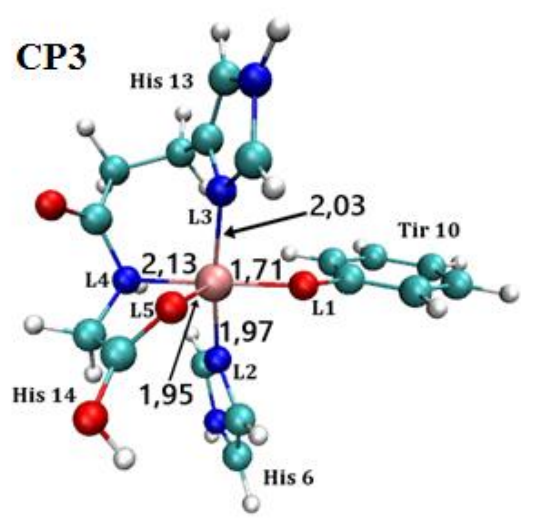

CT2

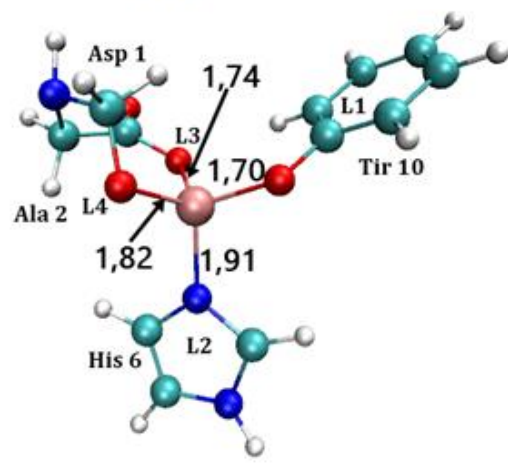

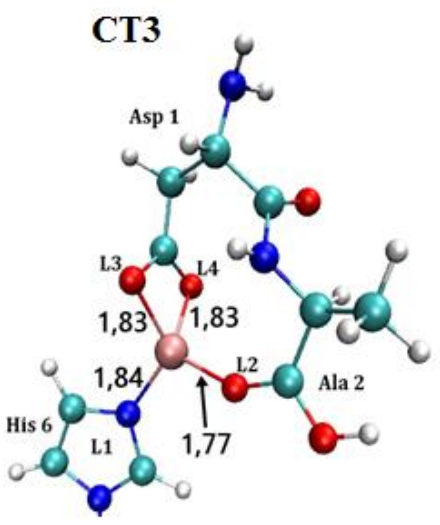

Fig. 3. Geometrías optimizadas de los complejos Al(III)-ßAA, distancia en Ángstrom (Á)

Ahora bien, con el propósito de estudiar la naturaleza de las interacciones $\mathrm{Al}(\mathrm{III})-\beta \mathrm{A}$, se utilizaron el índice de enlace de Wiberg $(\varphi)$, y el cambio en la carga de aluminio durante la interacción. Para ello, se hizo uso de la ecuación 3:

$$
\Delta Q_{\mathrm{Al}}=Q_{\mathrm{Al}}^{\mathrm{C}}-\mathrm{Q}_{\mathrm{Al}}
$$

Donde $Q^{c}{ }_{A l}$ representa la carga del aluminio cuando está presente en determinado complejo y $Q_{A l}$ es la carga del átomo de aluminio aislado. Ambos parámetros se calcularon usando el esquema NBO. En la tabla 2, se presenta el índice de enlace de Wiberg $(\varphi)$ para cada tipo de interacción.

Tabla 2: Índice de enlace de Wiberg $(\varphi)$ para cada tipo de interacción

\begin{tabular}{|c|c|c|c|c|c|c|}
\hline Complejo & $\operatorname{ImN}$ & $\mathrm{Ph}^{-}$ & $\mathrm{C}=\mathrm{O}$ & $\mathrm{COO}^{-}$ & $\mathrm{RN}$ & $\Delta \mathrm{Q}_{\text {AI }}$ \\
\hline CP3 & 0,310 & 0,440 & 0,240 & - & 0,220 & $-0,980$ \\
\hline CP1 & 0,310 & 0,440 & 0,260 & - & 0,260 & $-0,990$ \\
\hline CP2 & 0,340 & 0,380 & - & 0,270 & - & $-0,970$ \\
\hline CT3 & 0,400 & - & 0,340 & 0,320 & - & $-0,880$ \\
\hline CT2 & 0,320 & 0,450 & 0,300 & 0,380 & - & $-0,900$ \\
\hline CT1 & 0,320 & 0,410 & - & - & 0,260 & $-0,940$ \\
\hline
\end{tabular}

Las interacciones más fuertes se presentan entre el ligando $\mathrm{Ph}^{-} \mathrm{O}^{-}$y el ion aluminio, debido a que en aquellos complejos donde hay interacciones tipo $\mathrm{Ph}-\mathrm{O}^{-}$, el índice de enlace de Wiberg exhibe valores más altos respecto a otro tipo de interacciones. En general, se observa que las uniones con residuos de tirosina son 
más fuertes en complejos CP que en CT. Por otro lado, las interacciones ImN propias de los residuos de histidina son comunes en todos los complejos bajo estudio, y su fortaleza es mayor en los complejos tetracoordinados respecto a los penta-coordiados. El orden en los tipos de interacción respecto a su fortaleza fue determinado mediante el índice de enlace de Wiberg, obteniéndose la siguiente tendencia $\mathrm{Ph}-\mathrm{O}^{-}>\operatorname{ImN}>$ $\mathrm{COO}^{-}>\mathrm{C}=\mathrm{O}>\mathrm{RN}$, donde se observa que las interacciones $\mathrm{C}=\mathrm{O}$ y $\mathrm{RN}$ son poco frecuentes en los complejos presentados y su fortaleza de enlace es menor en comparación con las interacciones donde participan los grupos - $\mathrm{R}\left(\mathrm{PhO}^{-}, \mathrm{ImN}\right.$ y $\left.\mathrm{COO}\right)$ de los residuos del péptido $\beta A$. Lo anterior sugiere que durante la agregación de $\beta A$, el ion $\mathrm{Al}(\mathrm{III})$ tiende a formar interacciones principalmente con estos grupos - $\mathrm{R}$.

En cuanto a los efectos que producen estas uniones sobre el ion central $\mathrm{Al}(\mathrm{III}))$. De acuerdo con los valores de $\Delta Q_{A l}$, el cual mide el cambio que experimenta la carga del ion al formar interacciones con los aminoácidos, se observa que los complejos penta-coordinados inducen un cambio mayor en la carga de aluminio en comparación con los complejos tetra-coordinados (tabla 2). Se encontró que en el complejo CT3 donde la interacción tipo $\mathrm{PhO}^{-}$no se presenta, el cambio en la carga de $\mathrm{Al}(\mathrm{III})$ es menor, con respecto a los demás valores de $\Delta Q_{A l}$, sugiriendo que estas interacciones favorecen la transferencia de carga entre las especies químicas. Además, es bien sabido que los efectos producidos por la transferencia de carga, en este caso $\mathrm{Al}(\mathrm{III})$-ligando ( $\mathrm{Al}(\mathrm{III})$-L), están acompañados por cambios geométricos y energéticos en la esfera de coordinación de dichos complejos.

En la figura 4, se muestra la relación que guarda el índice de enlace de Wiberg $(\varphi)$, (calculado sobre cada

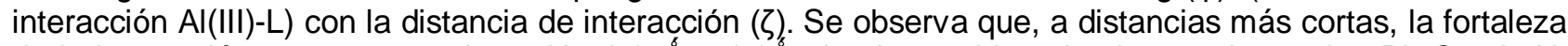
de la interacción es mayor, en la región $1,65 \AA$ a $1,8 \AA$, donde se ubican las interacciones tipo Ph-O e $\operatorname{ImN}$, discutidas anteriormente. Por otro lado, las interacciones tipo $\mathrm{RN}, \mathrm{C}=\mathrm{O}$ y $\mathrm{COO}^{-}$con distancias por encima de los $1,9 \AA$ p presentan valores más bajos de $\varphi$.

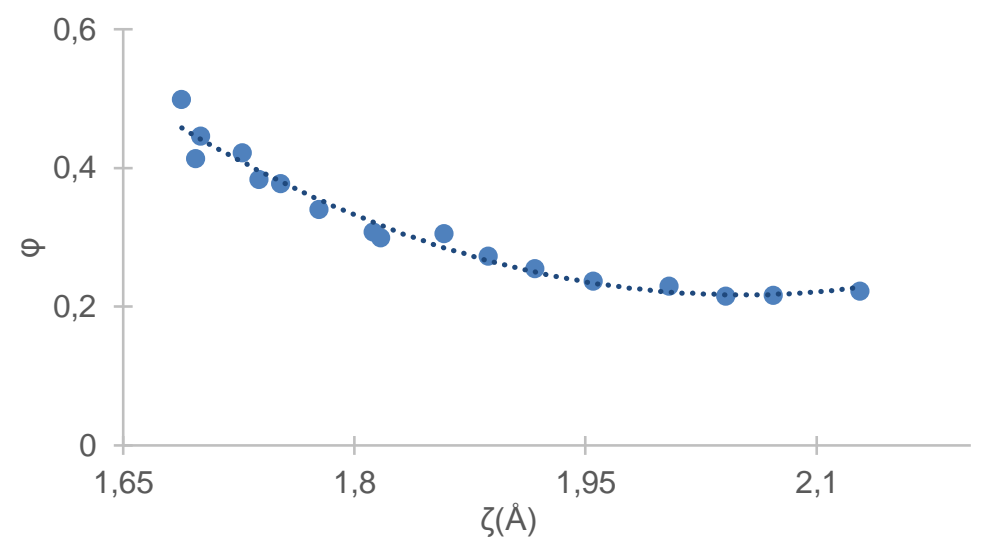

Fig. 4. Gráfico de $\varphi$ vs $\zeta$

De este modo, la proximidad de los aminoácidos del péptido $\beta A$ con el ion aluminio, juegan un papel importante en la formación de interacciones fuertes, sin embargo, también se hace necesario tener en cuenta los cambios conformacionales a los que debe someterse el péptido para formar este tipo de interacciones, por lo que la estabilidad termodinámica de los complejos formados puede dar información sobre este comportamiento. De ahí que, en la tabla 3 , se muestran los valores de $\Delta \mathrm{G}$ calculados para los complejos CT y CP. Se observa que los compuestos penta-coordinados son más estables que los tetra-coordinados, sin embargo, CT1 presenta mayor estabilidad que CP3. Cabe resaltar, que los aminoácidos Tyr10, His14, e His13, se encuentran presentes en los complejos con mayor estabilidad, lo cual apoya la idea de que estos aminoácidos son los principales centros de unión entre el péptido $\beta A$ y los metales (Mujika et al., 2017; Strodel y Coskuner-Weber, 2019). Por otro lado, el complejo CT3 presenta un valor positivo de $\Delta G$, lo cual se asocia a la usencia de interacciones tipo $\mathrm{Ph}^{-} \mathrm{O}^{-}$que estabilicen el complejo.

Tabla 3: Valores de energía libre calculados para los compuestos bajo estudio

\begin{tabular}{|c|c|}
\hline Complejo & $\Delta \mathrm{G}\left(\mathrm{kcal} \mathrm{mol}^{-1}\right)$ \\
\hline CP2 & $-1155,880$ \\
\hline CP1 & $-1012,740$ \\
\hline CT1 & $-953,850$ \\
\hline CP3 & $-938,140$ \\
\hline CT2 & $-513,480$ \\
\hline CT3 & 3625,690 \\
\hline
\end{tabular}




\section{CONCLUSIONES}

Se estudiaron computacionalmente las propiedades geométricas, electrónicas y termodinámicas de un grupo de complejos formados por Al(III) y los aminoácidos Asp1, Ala2, Glu3, His6, Tyr10, Glu11, His13, His14 que hacen parte de la región de afinidad por los metales del péptido $\beta$-Amiloide(1-16) implicado en la enfermedad de Alzheimer. De acuerdo a los resultados de este estudio y de su discusión, de la comparación con resultados de otros autores y del detallado análisis presentado se pueden extraer las siguiente cinco conclusiones principales:

1) Se encontró a la luz de los cálculos computacionales que el ion $\mathrm{Al}(\mathrm{III})$ forma complejos tetra-coordinados y penta-coordinados de forma estable con los grupos -R de los aminoácidos Asp1, Ala2, Glu3, His6, Tyr10, Glu11, His13, e His14, lo cual mostró buen acuerdo con resultados de trabajos teóricos previos.

2) Los funcionales B3LYP y M06-2X con la base $6-311+G(d, p)$ fueron de utilidad para estudiar las interacciones $\mathrm{Al}$ (III)-AA. Por otro lado, los complejos CT y CP mostraron interacciones con los átomos $\mathrm{O}$ y $\mathrm{N}$, de los ligandos $\mathrm{Ph}-\mathrm{O}, \mathrm{COO}, \mathrm{C}=\mathrm{O}, \mathrm{RN}$ e $\mathrm{ImN}$, respectivamente, donde las distancias de interacción fueron menores en $\mathrm{Ph}^{-} \mathrm{O}^{-}$e $\mathrm{ImN}$, ocurriendo el caso contrario con las interacciones $\mathrm{COO}^{-}, \mathrm{C}=\mathrm{O}$ y $\mathrm{RN}$, ya que mostraron distancias mayores.

3) Los valores de $\varphi$ mostraron que las interacciones más fuertes fueron $\mathrm{Ph}-\mathrm{O}-\mathrm{Al}(\mathrm{III})$ y ImN-Al(II). En general, se obtuvo que el tipo de interacciones según su fortaleza exhibió el orden $\mathrm{Ph}-\mathrm{O}^{-}>\operatorname{lmN}>\mathrm{COO}^{-}>\mathrm{C}=\mathrm{O}>\mathrm{RN}$, y en consecuencia los aminoácidos Tyr10, His13, His4 participan en la agregación del péptido $\beta A$. Adicionalmente, se observó que la distancia de estas interacciones se relaciona de forma inversa con su fortaleza de enlace, por lo que esta idea apoya que las interacciones Ph-O-Al(III) y ImN-Al(III) sean las más fuertes.

4) El análisis termodinámico de los complejos estudiados mostró que los complejos penta-coordinados son más estables que los tetra-coordinados (orden de estabilidad CP2>CP1>CT1>CP3>CT2>CT1). El complejo CT1 presentó un valor positivo de $\Delta \mathrm{G}$.

5) Los resultados obtenidos en este estudio apoyan la idea de que los aminoácidos de la región (1-16) del péptido $\beta A$ que presentan grupos $-R$ ionizables están directamente involucrados en la asociación de este péptido con los iones metálicos; principalmente los residuos Tyr10, His13, His14, indicando también que estos sitios pueden ser objetivo de estudio para el desarrollo de compuestos que inhiban la agregación acelerada de $\beta A$.

\section{AGRADECIMIENTOS}

Los autores agradecen a la Universidad de Córdoba, por el apoyo económico brindado para la realización de esta investigación.

\section{REFERENCIAS}

Alí-Torres, J., Mirats, A., Maréchal, J.D., Rodríguez-Santiago, L., y Sodupe, M., Modeling Cu2+-A $\beta$ complexes from computational approaches, AIP Advances, 5(9), 092402 (2015).

Bonda, D.J., Lee, H., Blair, J.A., Zhu, X., Perry, G., y Smith, M.A., Role of metal dyshomeostasis in Alzheimer's disease, Metallomics, 3(3), 267(2011).

Brookmeyer, R., Johnson, E., Ziegler-Graham, K., y Arrighi, H.M., Forecasting the global burden of Alzheimer's disease, Alzheimers Dement, 3(3), 186-191 (2007).

Bush, A., The metallobiology of Alzheimer's disease, Trends Neurosci, 26(4), 207-214 (2003).

Christen, Y., Oxidative processes in Alzheimer's disease: The role of A $\beta$-metal interactions, Exp Gerontol, 35(4), 445-451 (2000).

CSerpell, L. Alzheimer's amyloid fibrils: Structure and assembly, Biochim. Biophys. Acta-Mol. Bas. Dis, 1502(1), 16-30 (2000).

Chen, Y.S., Chen, S.D., Wu, C.L., Huang, S.S., y Yang, D.I., Induction of sestrin2 as an endogenous protective mechanism against amyloid beta-peptide neurotoxicity in primary cortical culture, Exp Neurol, 253, 63-71 (2014).

Drew, S.C., y Barnham, K.J., The Heterogeneous Nature of Cu2+ Interactions with Alzheimer's Amyloid $-\beta$ Peptide, Acc. Chem. Res. 44(11), 1146-1155 (2011).

Exley, C., Price, N.C., Kelly, S.M., y Birchall, J.D., An interaction of beta-amyloid with aluminium in vitro, FEBS. Lett. 324, 293-295 (1993). 
Figueredo, S.F., Ensuncho, A., y López, J., Exploración estocástica de las superficies de energía potencial de dímeros cis-trans y trans-trans del ácido fórmico, Quím. Nov, 37(8), 1365-1370 (2014).

Frisch, M., Trucks, G., y Schlegel, H., Gaussian 03, rev. B. 03. Gaussian Inc, Pittsburgh, PA (2003).

Hanwell, M.D., y otros cinco autores, Avogadro: An advanced semantic chemical editor, visualization and analysis platform, J. Cheminformatics, 4, 1-17(2012).

Harrington, C.R., y otros cuatro autores, Alzheimer's-disease-like changes in tau protein processing: Association with aluminium accumulation in brains of renal dialysis patients, The Lancet, 343(8904), 993-997 (1994).

Kepp, K., Alzheimer's disease: how metal ions define $\beta$-amyloid function, Coord. Chem. Rev, 351, 127-159 (2017).

Kohn, W., Becke, A.D., y Parr, R.G., Density Functional Theory of Electronic Structure, J. Phys. Chem, 100(31), 1297412980 (1996).

Lopes, J.F., Rocha, W.R., Dos Santos, H.F., y De Almeida, W.B., An investigation of the BSSE effect on the evaluation of Ab Initio interaction energies for cisplatin-water complexes, J. Braz. Chem. Soc. 21(5), 887-896 (2010).

Mottishaw, J. D., Erck, A.R., Kramer, J.H., Sun, H., y Koppang, M., Electrostatic Potential Maps and Natural Bond Orbital Analysis: Visualization and Conceptualization of Reactivity in Sangers Reagent, J. Chem. Educ, 92(11), 1846-1852 (2015).

Mujika, J.I., y otros cinco autores, Pro-oxidant activity of aluminum: stabilization of the aluminum superoxide radical ion, J. Phys. Chem. A 115, 6717-6723 (2011).

Mujika, J.I., y otros seis autores, Aluminium in biological environments: a computational approach, Comput. Struct. Biotechnol. J. 9, e201403002 (2014).

Mujika J.I., y otros seis autores, Elucidating the $3 D$ structures of $A((I I)-A \beta$ complexes: a template free strategy based on the pre-organization hypothesis, Chem. Sci. 8, 5041-5049 (2017).

Mujika J.I., Dalla Torre, G., y otros cinco autores, Aluminum's preferential binding site in proteins: sidechain of amino acids versus backbone interactions, J. Inorg. Biochem, 181, 11-116 (2018).

Nakano, Y. y otros seis autores, Long-Term Efficacy of Galantamine in Alzheimer's Disease: The Okayama Galantamine Study (OGS), JAD, 47(3), 609-617(2015).

Provias, J. y Jeynes, B., The role of the blood-brain barrier in the pathogenesis of senile plaques in Alzheimer's disease, Int J Alzheimers Dis, 2014, 1-7(2014).

Platts, J.A., Quantum chemical molecular dynamics and metadynamics simulation of aluminium binding to amyloid- $\beta$ and related peptides, R. Soc. Open. Sci, 7, 191562 (2020).

Rauk, A., The chemistry of Alzheimer's disease, Chem. Soc. Rev, 38(9), 2698 (2009).

Swaddle, T.W., Rosenqvist, J., Yu, P., Bylaska, E., Phillips, B.L., y Casey, W.H., Kinetic Evidence for Five-Coordination in $A / O H(a q)^{2+}$ Ion, Science, 308, 1450-1453 (2005).

Silvia, B., Messori, L., Drago, D., Gabbiani, Ch., Cendron, L., y Zatta, P., Aluminum, copper, iron and zinc differentially alter amyloid-Aß1-42 aggregation and toxicity, Int. J. Biochem. Cell Biol, 43(6), 877-885 (2011).

Song, B., y otros once autores, Irreversible Denaturation of Proteins through Aluminum-Induced Formation of Backbone Ring Structures, Angew. Chem. Int. Ed, 53, 6358-6363 (2014).

Strodel, B., y Coskuner-Weber, O., Transition metal ion interactions with disordered amyloid- $\beta$ peptides in the pathogenesis of Alzheimer's disease: insights from computational chemistry studies, J. Chem. Inf. Model. 59, 1782-1805 (2019).

Slater, J.C., A Simplification of the Hartree-Fock Method, Phys. Rev, 81(3), 385-390 (1951).

Thaís, T., y otros cuatro autores, Molecular structure of tetraaqua adenosine 5'-triphosphate aluminium(III) complex: $A$ study involving Raman spectroscopy, theoretical DFT and potentiometry, Spectrochim. Acta A, 105, 88-101 (2013)

Tokutake, S., Nagase, H., Morisaki, S., y Oyanagi, S., Aluminium detected in senile plaques and neurofibrillary tangles is contained in lipofuscin granules with silicon, probably as aluminosilicate, Neurosci. Lett, 185(2), 99-102 (1995).

Tõugu, V., Tiiman, A., y Palumaa, P., Interactions of Zn(II) and Cu(II) ions with Alzheimer's amyloid-beta peptide. Metal ion binding, contribution to fibrillization and toxicity, Metallomics, 3(3), 250 (2011).

Weintraub, S., Wicklund, A.H., y Salmon, D.P., The Neuropsychological Profile of Alzheimer Disease. Cold. Spring. Harb. Perspect. Med, 2(4), 1-18 (2012).

Yang, W., Qian, Z., Miao, Q., Wang, Y. y Bi, S., Density functional theory study of the aluminium(III) hydrolysis in aqueous solution, Phys. Chem. Chem. Phys., 11, 2396-401 (2009).

Zhang, Q., Zhang, F., Ni, Y., y Kokot, S., Effects of aluminum on amyloid-beta aggregation in the context of Alzheimer's disease, Arab. J. Chem, 12(8), 2897-2904 (2019). 